CASO CLÍNICO

\title{
Prematuro con perforación intestinal espontánea de presentación muy precoz
}

\author{
Jorge L. Alvarado-Socarras ${ }^{1 *}$, Andrea Gómez-Capacho ${ }^{1}$, Maria A. Niño-Tovar ${ }^{1}$ y Adriana Pinilla-Orejana ${ }^{2}$ \\ ${ }^{1}$ Unidad Neonatal, Fundación Cardiovascular de Colombia, Hospital Internacional de Colombia; ${ }^{2}$ Departamento de Cirugía Pediátrica, Fundación \\ Cardiovascular de Colombia. Bucaramanga, Colombia
}

\begin{abstract}
Resumen
Introducción: La perforación intestinal espontánea es una condición que afecta principalmente a neonatos pretérmino. Caso clínico: Se presenta el caso de un neonato pretérmino de 26 semanas de gestación con neumoperitoneo en las primeras 24 horas de vida. Por lo precoz del cuadro y los hallazgos radiológicos, la primera hipótesis diagnóstica fue una perforación gástrica. Sin embargo, en la laparotomía se encontró una perforación ileal única, sin hallazgos de enterocolitis. Conclusiones: La perforación intestinal espontánea se ha asociado con intervenciones médicas, como el uso concomitante de esteroides e indometacina. Otras intervenciones, como el uso de magnesio prenatal y la colocación de surfactante pulmonar de forma mínimamente invasiva, se han propuesto como factores de riesgo para su desarrollo. En la actualidad, deben evaluarse la perforación intestinal espontánea y sus asociaciones. Las presentes y futuras investigaciones podrían contribuir a aclarar el papel de las intervenciones mencionadas en la revisión preliminar de la literatura.
\end{abstract}

Palabras clave: Perforación intestinal espontánea. Sulfato de magnesio. Enterocolitis necrosante. Prematuro.

\section{Preterm neonate with spontaneous intestinal perforation of very early presentation}

\section{Abstract}

Background: Spontaneous intestinal perforation is a condition that mainly affects preterm infants. Case report: The case of a preterm infant of 26 weeks of gestation with pneumoperitoneum in the first 24 hours of life is described. Due to the early symptoms and radiological findings, the first diagnostic hypothesis was gastric perforation. However, at laparotomy, a unique ileal perforation was found, without findings of enterocolitis. Conclusions: Spontaneous intestinal perforation is a complication associated with medical interventions such as the concomitant use of steroids and indomethacin. Recently, other interventions, such as the use of prenatal magnesium and treatment with exogenous pulmonary surfactants in a minimally invasive way have been proposed as risk factors for its development. At present, clinicians should evaluate spontaneous intestinal perforation and its associations, and ongoing and future research may clarify the role of the interventions mentioned in the review of preliminary literature.

Key words: Spontaneous intestinal perforation. Magnesium sulfate. Necrotizing enterocolitis. Preterm.

Correspondencia:

*Jorge L. Alvarado-Socarras

E-mail: jorgealso2@yahoo.com
Fecha de recepción: 11-03-2020

Fecha de aceptación: 04-05-2020

DOI: 10.24875/BMHIM.20000054
Disponible en internet: 09-09-2020

Bol Med Hosp Infant Mex. 2021;78(2):143-147

www.bmhim.com 1665-1146/@ 2020 Hospital Infantil de México Federico Gómez. Publicado por Permanyer. Este es un artículo open access bajo la licencia CC BY-NC-ND (http://creativecommons.org/licenses/by-nc-nd/4.0/). 


\section{Introducción}

La perforación intestinal espontanea (PIE) fue descrita en 1988 y se define por la presencia de una perforación, habitualmente en el intestino delgado y sobre todo en el íleon terminal, sin inflamación. Aunque la etiología es desconocida, se ha asociado con el uso de presión positiva, indometacina y dexametasona. Es una condición diferente de la enterocolitis necrosante $(E C N)$, pero se relaciona con acidosis metabólica, hipotensión y sepsis. Estos desenlaces se han asociado con leucomalacia periventricular y retraso del neurodesarrollo'. La PIE se puede presentar a cualquier edad gestacional; sin embargo, se relaciona con más frecuencia con prematuridad extrema, lo que de por sí ya representa un factor de riesgo para la discapacidad neurológica². Por ello, la PIE se convierte en un marcador con impacto negativo en los prematuros que la sufren.

La edad promedio de presentación de la PIE en prematuros es alrededor del séptimo día de vida, un poco más temprano que la $\mathrm{ECN}^{3}$.

Se presenta un caso de PIE de forma muy temprana, sin sospecha clínica inicial por las condiciones perinatales asociadas.

\section{Caso clínico}

Se trata de un pretérmino de sexo femenino y 26 semanas de gestación (SDG), y parto por síndrome HELLP (hemolysis, elevated liver enzymes, low platelet count). La madre recibió maduración pulmonar, alfa-metildopa, nifedipino y sulfato de magnesio previamente a la cesárea. Se obtuvo pretérmino de $510 \mathrm{~g}$, talla $36 \mathrm{~cm}$, Apgar de 9 y 9 , que presentó síndrome de dificultad respiratoria precoz, por lo que se inició soporte con pieza en T (Neopuff $\left.{ }^{\circledR}\right)\left(5 \mathrm{cmH}_{2} \mathrm{O} / \mathrm{FiO}_{2} 30 \%\right)$. La paciente fue trasladada a la unidad de cuidados intensivos neonatales, donde se inició soporte con presión positiva continua en la vía aérea (CPAP). Presentó deterioro del patrón respiratorio, por lo que requirió intubación y colocación de surfactante pulmonar a las 6 horas de vida, además de catéteres umbilicales. El control radiológico a las 5 horas del procedimiento evidenció la presencia de neumoperitoneo (a las 11 horas de vida). Los análisis de laboratorio iniciales, como hemograma, proteína $C$ reactiva y niveles de magnesio, fueron normales. La sospecha clínica inicial fue perforación gástrica debido a la posición de la sonda orogástrica (Figura 1). Se inició tratamiento antibiótico con ampicilina, gentamicina y metronidazol. Debido al bajo peso, se decidió colocar un drenaje peritoneal en el hemiabdomen izquierdo.

La paciente presentó mejoría de su condición hemodinámica y respiratoria en las primeras 72 horas. Posteriormente se observó un aumento del drenaje de contenido intestinal, por lo cual se realizó una laparotomía exploratoria y se encontró una perforación intestinal única en el íleon terminal sin signos de enterocolitis, por lo que se realizó resección intestinal de $1 \mathrm{~cm}$ más ileostomía. La muestra fue enviada a patología (Figura $2 \mathrm{~A}$ y B). Se analizó el cultivo de líquido peritoneal, cuyos resultados no fueron clínicamente relevantes. Se realizó ecocardiograma a las 72 horas, sin hallazgos de conducto arterioso ni de otra malformación estructural. La ecografía cerebral no mostró sangrado en las semanas 1,2 y 3 .

La paciente permaneció críticamente enferma durante 70 días; requirió soporte vasoactivo y antibióticos de amplio espectro, con único aislamiento de Staphylococcus epidermidis resistente a la meticilina en los hemocultivos y el cultivo de la punta de los catéteres centrales, lo que dificultó su control.

Se logró el control de la sepsis, se realizó la extubación electiva y se administró soporte enteral progresivo, hasta lograr la recuperación nutricional. Después de 104 días de estancia hospitalaria, se realizaron estudios de tránsito intestinal y colon por enema, sin evidencia de zona de estenosis, por lo que se realizó el cierre de la ileostomía sin complicaciones. Finalmente, la paciente egresó con un peso de $2,750 \mathrm{~g} \mathrm{y}$ recibiendo alimentación por succión.

\section{Discusión}

El neumoperitoneo es una condición grave con alto riesgo de mortalidad. Aunque sus causas son múltiples, la principal es la presentación secundaria a ECN, cuyas diferencias clínicas, anatómicas, de prevalencia y pronóstico en comparación con la PIE se muestran en la Tabla 1. Sin embargo, otras condiciones han sido reportadas como causa de neumoperitoneo, como trauma por sonda de alimentación, estimulación anal con termómetros rectales, obstrucción intestinal y $\mathrm{PIE}^{3,4}$. Esta última es una condición que se presenta con más frecuencia en neonatos pretérmino, principalmente de menos de 1,000 g de peso, y es la segunda causa de neumoperitoneo. Algunos factores, como hipoxia, choque, Apgar bajo, rotura de membranas o reanimación en el periodo perinatal pueden predisponer a la $\mathrm{PIE}^{5}$. En este grupo suele observarse después de los primeros 3 días de vida. Aquellos de presentación temprana 
Tabla 1. Diferencias entre perforación por enterocolitis necrosante (ECN) y perforación intestinal espontánea (PIE) $)^{6,7}$

\begin{tabular}{|l|l|l|}
\hline & ECN & PIE \\
\hline Inicio & Precedido de signos clínicos & Súbito en general \\
\hline Mortalidad & Mayor & Menor \\
\hline Tiempo de presentación & Pico a las 30-32 semanas de gestación corregida & Temprano (7 días), incluso in utero \\
\hline Características clínicas & $\begin{array}{l}\text { Signos previos de distensión abdominal, isquemia } \\
\text { intestinal y neumatosis }\end{array}$ & $\begin{array}{l}\text { Coloración azul de pared abdominal, disminución de } \\
\text { gas intestinal sin neumatosis }\end{array}$ \\
\hline Factores relacionados & Microbiota, leche materna, antiácidos & No relacionado \\
\hline
\end{tabular}

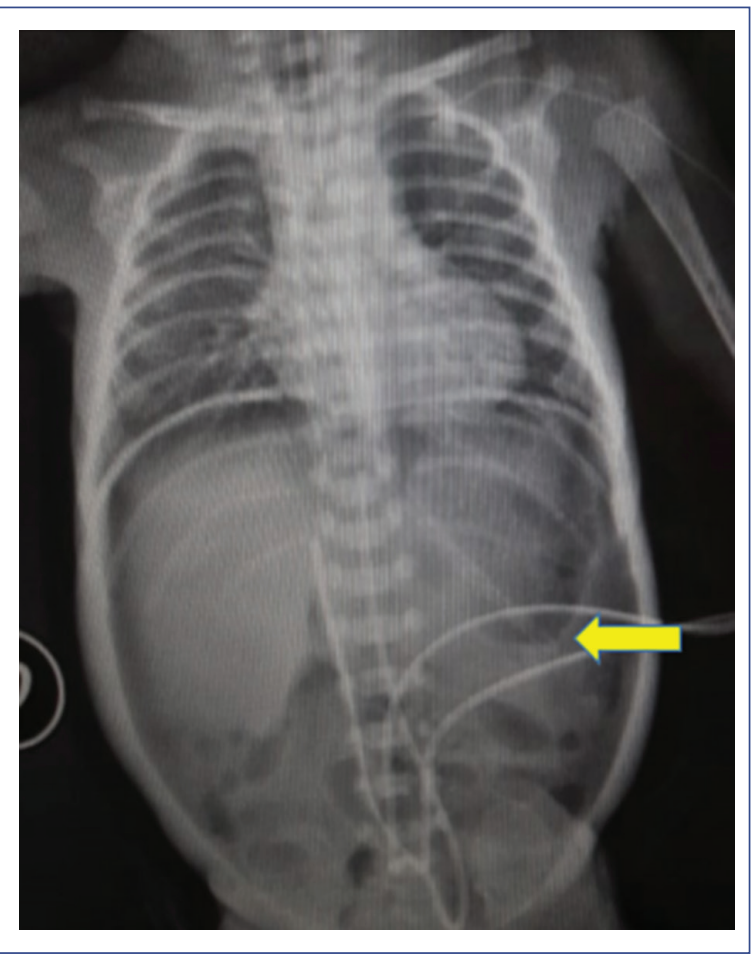

Figura 1. Neumoperitoneo. Sonda orogástrica inserta (flecha), por lo cual se sospechó perforación gástrica como causa del neumoperitoneo.

son prematuros menos inmaduros, con un peso de alrededor de 1,400 g. Sin embargo, no es una condición exclusiva de la prematuridad, y en neonatos a término y casi a término la presentación es más temprana ${ }^{3,6,7}$. Por estos datos, en el presente caso no se sospechó inicialmente la PIE.

Algunos otros factores relacionados con la PIE son el uso posnatal temprano de esteroides (< 48 horas), el uso de indometacina y los eventos de asfixia perinatal. Incluso se ha reportado la CPAP como factor de riesgo. Algunas terapias empleadas en prematuros pueden ejercer un efecto sumatorio en el desarrollo de $\mathrm{PIE}^{6}$, como el empleo concomitante de esteroides e indometacina.

Como factores de riesgo para neumoperitoneo se han postulado algunas alteraciones anatómicas de la pared intestinal, como la ausencia de la capa muscular externa, condición que no ha podido ser probada a la fecha, y las condiciones perinatales y la prematuridad, que prevalecen como principales factores de riesgo?. Otros factores asociados son las infecciones por $S$. epidermidis y por cándida.

En este caso se reporta PIE posnatal y aislamiento en los hemocultivos de $S$. epidermidis, que es considerada por algunos como una bacteria oportunista y no como causante de la patología ${ }^{5,6}$. Por otro lado, en estudios de placenta se ha aislado cándida en casos de PIE. De ahí la importancia del estudio anatomopatológico de la placenta en los pacientes de alto riesgo?.

El principal hallazgo radiológico de la PIE es el neumoperitoneo, el cual es rápidamente progresivo ya que genera gran compromiso cardiopulmonar, por lo que es la principal indicación de intervención quirúrgica ${ }^{6}$. Los pacientes afectados lo presentan, en general, poco tiempo después del inicio de la vía oral, con distensión súbita y, en ocasiones, asociado con una coloración azulada de la pared abdominal. Sin embargo, existen pocos reportes de presentación de PIE en las primeras horas de vida, aunque se han reportado series de casos que informan de un rango generalmente después del primer día ${ }^{8}$.

Por lo precoz de la presentación y los hallazgos radiológicos anotados en este caso, se sospechó una perforación gástrica traumática como etiología del neumoperitoneo ${ }^{3}$.

Por la prematuridad extrema, el manejo que se llevó a cabo fue la colocación transitoria de un drenaje peritoneal (el cual ha sido establecido en caso de neumoperitoneo secundario a ECN o PIE) hasta la estabilidad de la paciente. Incluso, el drenaje se ha indicado en caso de 


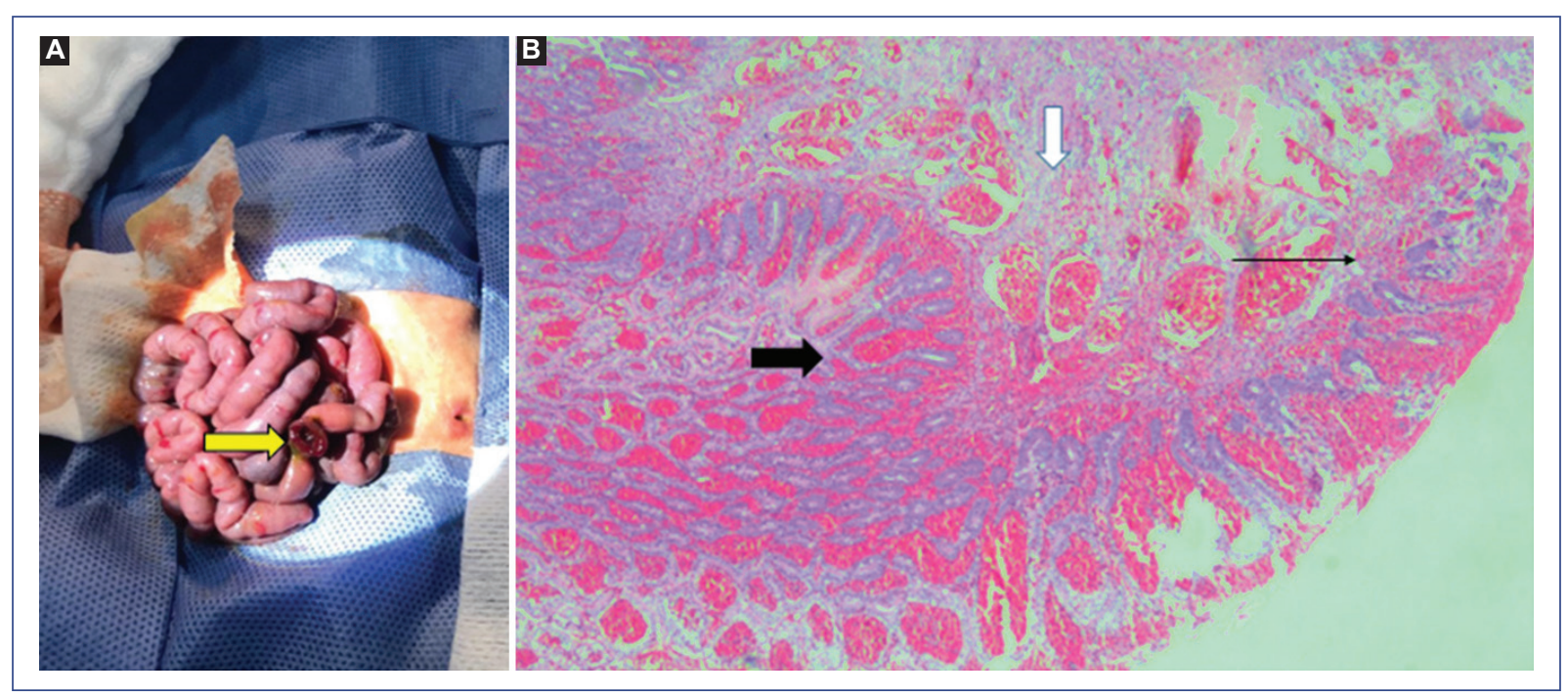

Figura 2. A: Perforación ileal única, sin cambios inflamatorios. Algunas lesiones rojas en las asas corresponden a la manipulación durante el procedimiento. Hallazgos macroscópicos confirmados con informe patológico. B: Íleon de arquitectura conservada con hemorragia reciente en la submucosa (flecha negra gruesa) y congestión vascular en los vasos de la subserosa (flecha blanca), con cambios por isquemia (flecha negra fina). No se reconoce componente inflamatorio en el tejido evaluado.

perforación gástrica secundaria a colocación de sonda orogástrica, con resultados favorables hasta en el $85 \%{ }^{9}$.

Por otro lado, someter a neonatos prematuros a procedimientos invasivos de tipo anestésico y quirúrgico de forma temprana podría relacionarse con mayores riesgos, como hemorragia intraventricular, con pobres resultados neurológicos. El manejo conservador mediante un drenaje peritoneal puede ser definitivo en algunos casos, pero la persistencia de neumoperitoneo, acidosis y evidencia de peritonitis determina la exploración quirúrgica. En este caso, se determinó la intervención ${ }^{10,11}$. Algunas condiciones clínicas radiológicas, como una mínima distensión, signos de buena perfusión sistémica, mínimo líquido libre o aire en la cavidad peritoneal, y plaquetas normales, entre otros, podrían definir el éxito del manejo conservador ${ }^{4}$. A pesar de estas alternativas, el manejo debe ser interdisciplinario, incluyendo neonatólogos y cirujanos pediatras, dadas las condiciones y decisiones cambiantes que ameritan diferentes abordajes según la evolución clínica. Además de las intervenciones, drenaje o cirugía, el reposo intestinal, el uso de antibióticos, la nutrición parenteral, la sonda orogástrica descompresiva y, en ocasiones, el soporte vasoactivo complementan el manejo en estos pacientes ${ }^{6}$.

En pretérminos extremos se han propuesto algunas intervenciones para disminuir la morbilidad neurológica y respiratoria. Una de ellas es el potencial efecto neuroprotector del sulfato de magnesio en prematuros. De ahí que su uso se haya generalizado como práctica para disminuir el riesgo de parálisis cerebral (nivel de evidencia A2) ${ }^{12}$, sin mencionar los beneficios en las gestantes con preeclampsia. Sin embargo, su implementación se ha planteado como un factor de riesgo de PIE, principalmente en pretérmino muy extremos $(<26$ $S D G)^{11}$. El magnesio atraviesa libremente la placenta, por lo que se ha planteado que altera el flujo mesentérico. Además, posee efecto colinérgico, lo que altera la motilidad intestinal, factor que se relaciona con posible daño intestinal ${ }^{13}$. No se ha detectado asociación con el tiempo de exposición ni con la dosis administrada de este elemento a la gestante; no obstante, podría existir una disminución de su depuración en el neonato prematuro, lo que ha sido planteado como un factor de riesgo ${ }^{14}$. A pesar de lo anterior, este efecto no ha sido demostrado y podría estar relacionado con el grado de inmadurez $z^{6,8,13}$.

El reto de la atención de los recién nacidos pretérmino cada vez más extremos (24-25 SDG) y con alto riesgo de discapacidad cognitiva y parálisis cerebral (inversamente proporcional a la edad gestacional), conduce a generalizar las intervenciones con un impacto favorable en estos desenlaces. Sin embargo, es probable que el verdadero impacto esté determinado por los subgrupos a intervenir, como la edad gestacional, y las condiciones materno-fetales, como 
la presencia de corioamnionitis, en quienes, al parecer, el beneficio en la reducción de la parálisis cerebral no sería tan efectivo ${ }^{13}$. Además, las intervenciones son un factor de estrés, con elevación de los niveles de cortisol fetal, lo que las convierte en un riesgo potencial de $\mathrm{PIE}^{6,7}$.

Otro serio problema es el abordaje de prematuros cada vez de menor edad gestacional y la alta incidencia persistente de displasia broncopulmonar, que se relaciona con procesos de intubación orotraqueal para la colocación de surfactante. Por esta razón empiezan a ser evaluados los métodos menos invasivos de aplicación del surfactante pulmonar. La aplicación del surfactante a través de un catéter en la luz traqueal (con respiración espontánea del prematuro para luego mantener un soporte ventilatorio no invasivo) está relacionada con una disminución de la necesidad de intubación en los primeros días, así como con una mejoría de la sobrevida libre de displasia broncopulmonar. El análisis de los trabajos realizados señala esta intervención como posible factor de riesgo para $\mathrm{PIE}^{15}$.

La PIE es una condición asociada fuertemente con la prematuridad, y debería evaluarse sistemáticamente en relación con las intervenciones perinatales utilizadas, las cuales buscan mejorar los resultados tempranos y tardíos en neonatos prematuros. A excepción de la prematuridad, ningún otro factor de riesgo ha sido demostrado, lo que obliga a realizar estudios de las diferentes intervenciones planteadas como factores de riesgo.

\section{Responsabilidades éticas}

Protección de personas y animales. Los autores declaran que para esta investigación no se han realizado experimentos en seres humanos ni en animales.

Confidencialidad de los datos. Los autores declaran que han seguido los protocolos de su centro de trabajo sobre la publicación de datos de pacientes.

Derecho a la privacidad y consentimiento informado. Los autores han obtenido el consentimiento informado de los pacientes o sujetos referidos en el artículo. Este documento obra en poder del autor de correspondencia.

\section{Conflicto de intereses}

Los autores declaran no tener ningún conflicto de intereses.

\section{Financiamiento}

\author{
Ninguno.
}

\section{Agradecimientos}

Al Departamento de Patología del Hospital Internacional de Colombia, Piedecuesta, Santander, Colombia. A los doctores Edwin Abraham Medina Medina y Johana Navarro Mejía.

\section{Bibliografía}

1. Rayyan M, Myatchin I, Naulaers G, Ali Said Y, Allegaert K, Miserez M. Risk factors for spontaneous localized intestinal perforation in the preterm infant. J Matern Fetal Neonatal Med. 2018;31:2617-23.

2. Shah J, Singhal N, da Silva O, Rouvinez-Bouali N, Seshia M, Lee SK, et al. Intestinal perforation in very preterm neonates: risk factors and outcomes. J Perinatol. 2015;35:595-600.

3. de Abreu Amaro C, Oliveira Pereira C, Mesquita J. Spontaneous intestinal perforation in a preterm neonate. BMJ Case Rep. 2018;11:1-2.

4. Khan RA, Mahajan JK, Rao KL. Spontaneous intestinal perforation in neonates: is surgery always indicated? Afr J Paediatr Surg. 2011; 8:249-51.

5. Tiwari C, Sandlas G, Jayaswal S, Shah H. Spontaneous intestinal perforation in neonates. J Neonatal Surg. 2015;4:1-4.

6. Kim ES, Brandt ML. Spontaneous intestinal perforation of the newborn. UpToDate; 2020. Disponible en: https://www.uptodate.com/contents/ spontaneous-intestinal-perforation-of-the-newborn.

7. Gordon PV, Attridge JT. Understanding clinical literature relevant to spontaneous intestinal perforations. Am J Perinatol. 2009;26:309-16.

8. Houben $\mathrm{CH}$, Feng XN, Chan KWE, Mou JWC, Tam YH, Lee KH. Spontaneous intestinal perforation: the long-term outcome. Eur J Pediatr Surg. 2017;27:346-51

9. Thanhaeuser M, Lindtner-Kreindler C, Berger A, Haiden N. Conservative treatment of iatrogenic perforations caused by gastric tubes in extremely low birth weight infants. Early Hum Dev. 2019;137:1-5.

10. Kshirsagar AY, Vasisth GO, Ahire MD, Kanojiya RK, Sulhyan SR. Acute spontaneous gastric perforation in neonates: a report of three cases. Afr J Paediatr Surg. 2011;8:79-81.

11. Broekaert I, Keller T, Schulten D, Hünseler C, Kribs A, Dübbers M. Peritoneal drainage in pneumoperitoneum in extremely low birth weight infants. Eur J Pediatr. 2018;177:853-8.

12. Sweet DG, Carnielli V, Greisen G, Hallman M, Ozek E, Te Pas A, et al. European consensus guidelines on the management of respiratory distress syndrome - 2019 update. Neonatology. 2019;115:432-50.

13. Garg BD. Antenatal magnesium sulfate is beneficial or harmful in very preterm and extremely preterm neonates: a new insight. $J$ Matern Fetal Neonatal Med. 2019;32:2084-90.

14. Mikhael M, Bronson C, Zhang L, Curran M, Rodriguez H, Bhakta KY. Lack of evidence for time or dose relationship between antenatal magnesium sulfate and intestinal injury in extremely preterm neonates. Neonatology. 2019;115:371-8.

15. Härtel C, Paul P, Hanke K, Humberg A, Kribs A, Mehler K, et al. Less invasive surfactant administration and complications of preterm birth. Sci Rep. 2018;8:1-7. 